\title{
Maximum thermodynamic power coefficient of a wind turbine
}

\author{
J. M. Tavares ${ }^{1,2}(\mathbb{D})$ P. Patrício ${ }^{1,2} \mathbb{D}$
}

${ }^{1}$ ISEL-Instituto Superior de Engenharia de Lisboa, Instituto Politécnico de Lisboa, Avenida Conselheiro Emído Navarro, Lisboa, Portugal ${ }^{2}$ Centro de Física Teórica e Computacional, Universidade de Lisboa, Lisboa, Portugal

\section{Correspondence}

J. M. Tavares, ISEL-Instituto Superior de Engenharia de Lisboa, Instituto Politécnico de Lisboa, Avenida Conselheiro Emído Navarro, Lisboa, Portugal.

Email: jose.tavares@isel.pt

Funding information

Fundação para a Ciência e a Tecnologia,

Grant/Award Number: UID/FIS/00618/2019

\begin{abstract}
According to the centenary Betz-Joukowsky law, the power extracted from a wind turbine in open flow cannot exceed $16 / 27$ of the wind transported kinetic energy rate. This limit is usually interpreted as an absolute theoretical upper bound for the power coefficient of all wind turbines, but it was derived in the special case of incompressible fluids. Following the same steps of Betz classical derivation, we model the turbine as an actuator disk in a one dimensional fluid flow but consider the general case of a compressible reversible fluid, such as air. In doing so, we are obliged to use not only the laws of mechanics but also and explicitly the laws of thermodynamics. We show that the power coefficient depends on the inlet wind Mach number $M_{0}$, and that its maximum value exceeds the Betz-Joukowsky limit. We have developed a series expansion for the maximum power coefficient in powers of the Mach number $M_{0}$ that unifies all the cases (compressible and incompressible) in the same simple expression: $\eta_{\max }=16 / 27+8 / 243 \mathrm{M}_{0}^{2}$.
\end{abstract}

\section{KEYWORDS}

Betz law, thermodynamics, wind turbines

\section{1 | INTRODUCTION}

The second law of thermodynamics sets limits to the power coefficient of all energy conversions. Knowledge of the maximum power coefficient of a given conversion process is crucial to increasing its performance. Thermodynamics establishes an hierarchy for the power coefficient $\eta$ of cyclic heat engines: (a) it is not possible to convert all heat into work, $\eta<1$; (b) the power coefficient cannot exceed that of a Carnot cycle between the higher, $T_{\max }$, and lower temperatures, $T_{\min }$, it attains, $\eta<1-T_{\min } / T_{\max }$, independently of other specificities of the engine; (c) the maximum power coefficient can be calculated for any specific set of cyclic transformations, if these are considered reversible and if the equations of state of the substance that operates the engine are known. ${ }^{1}$ Examples of the latter are well known for different cycles of an ideal gas that emulate several real engines ${ }^{2}$ : the Otto cycle (a gasoline engine), the Brayton cycle (a jet engine), the Diesel cycle (a diesel engine), etc.

Nowadays, heat engines are being replaced by renewable energy converters, the most rapidly growing of which are wind turbines for electricity generation. ${ }^{3}$ Wind turbines transform the kinetic energy of wind into work with no need for heat sources. The study of their power coefficient dates back to the 1920's when the so called Betz-Joukowsky (BJ) law was derived ${ }^{4,5}$ : by considering the turbine as a thin disc-or actuator-normal to a stationary, nonviscous and incompressible flow, the ratio between the power produced by the turbine and that carried by the fluid flow was shown to have a maximum of $16 / 27$. Although it was derived for incompressible flows, it is not uncommon to find references in the literature to the BJ limit such as "the maximum amount of energy one can get from the wind," 5 "the maximum theoretically possible rotor power coefficient," "theoretical maximum for an ideal wind turbine,"7 and "maximum achievable value of the power coefficient." 8 This suggests that this limit is being interpreted as a kind of Carnot limit: just like any cyclic heat engine has $\eta<1-T_{\min } / T_{\max }$, also any wind turbine would have $\eta<16 / 27$, independently of all its specificities.

The derivation of the BJ limit seems to rely only on the laws of mechanics (mass, momentum, and energy conservation). $6,9,10$ This is because this result was derived in the context of water propellers and then applied to wind turbines exposed to low velocity air flows ${ }^{5}$ : The incompressibility hypothesis, valid in these cases, leads to the decoupling of the mechanical and thermodynamical descriptions of the flow. The purpose of this work is to derive a more general expression for the maximum power coefficient of a wind turbine allowing for the finite compressibility of a fluid, such as air. By doing so, we are obliged to use not only the laws of mechanics but also and explicitly the laws of thermodynamics. We show that 
the power coefficient depends on the inlet wind Mach number $M_{0}$. While the $\mathrm{BJ}$ law is recovered in the limit of zero $M_{0}$, it will be shown that the famous limit it sets for power coefficient (16/27) can be surpassed if one considers, eg, isentropic or isothermal flows.

The actuator disk theory for compressible flows has been previously studied in the 1950s, in the context of propellers ${ }^{11,12}$ and more recently in the context of turbines. ${ }^{13}$ Our approach to the problem is very similar to those presented in these studies, and our final results for isentropic flows coincide with the numerical results of Oo. ${ }^{13}$ However, in our derivation, we explicitly use the second law of thermodynamics and put in evidence both its relation to the calculation of maximum efficiencies and the necessity to consider the entropy in compressible flows. This allows to treat all types of flow (compressible and incompressible) in a unified manner and to consider several types of compressible flows in a systematic way. As a consequence, we analyze not only the isentropic (like previous studies ${ }^{11-13}$ ) but also the isothermal case. Moreover, the final expressions for the efficiency in the compressible flows obtained here are simpler, more clear, and more easily related to the Betz limit than those of previous studies. ${ }^{11-13}$ This simplicity is manifest in the derivation of a novel series expansion for the maximum power coefficient in powers of the Mach number $M_{0}$ that unifies all the cases (compressible and incompressible): $\eta_{\max }=16 / 27+8 / 243 M_{0}^{2}$. For the isentropic flow, this expression is approximately valid up to $M_{0}<0.8$. In Section 3.5, we seek for a more detailed explanation to the increase in power coefficient, that we did not find in previous studies. ${ }^{11-13}$

\section{2 | POWER COEFFICIENT OF A WIND TURBINE}

The power coefficient $\eta$, or efficiency, ${ }^{4,9}$ of a wind turbine is defined as follows. ${ }^{5,6,9,14}$ Consider a fluid of mass density $\rho_{0}$ in a uniform, horizontal flow with velocity $c_{0}$. The kinetic energy per unit time (power) carried by the wind that crosses a vertical planar surface of area $A$ is $P_{w}=\frac{1}{2} \rho_{0} A c_{0}^{3}$. A turbine is placed in this surface, distorts the flow, and generates a power $\dot{W}_{t}$. Its power coefficient is then

$$
\eta=\frac{\dot{W}_{t}}{P_{w}},
$$

ie, the fraction of energy that the turbine can take from the flowing air and transform into work. The calculation of $\dot{W}_{t}$ is done by applying energy conservation to a control volume $(\mathrm{CV})$ that contains the turbine. ${ }^{9}$ The first law of thermodynamics applied to $\mathrm{CV}$ is

$$
\oiint_{C S}\left(h+\frac{c^{2}}{2}\right) \vec{\rho} \cdot \hat{n} d S=\dot{Q}-\dot{W}
$$

where CS is the boundary of $C V, \hat{n}$ is the outward-pointing normal to $C S, h, \rho$, and $\vec{c}$ are the enthalpy per unit mass, the mass density, and the velocity of the fluid, respectively. $\dot{Q}$ and $\dot{W}$ are the heat and the work per unit time exchanged by the fluid in $C V$ with its vicinity, respectively. The sign convention followed is such that $\dot{Q}<0$ and $\dot{W}>0$ correspond to energy leaving $C V$ to its vicinity. Notice that $h+\frac{c^{2}}{2}$ is the energy per unit mass transported by a fluid and that $\dot{W}$ represents all the types of work exchanged, ie, the power delivered by the turbine and the dissipative work of viscous forces. The second law of thermodynamics can be expressed through a generalized Clausius inequality ${ }^{15}$ (see Supporting Information) applied to CV

$$
\iiint_{C V} T \rho \vec{C} \cdot \nabla s d V \geq \dot{Q}
$$

where $\nabla s$ is the gradient of the entropy per unit mass of the fluid and $T$ is the temperature. The equality in (3) is valid for reversible processes, which, in the context of fluid flow, are obtained when viscous forces are absent and the transfer of heat originates by conduction only and associated to a well defined gradient of $T$. Combination of the first (2) and second laws of thermodynamics (3) results in an expression for the reversible power produced by the turbine,

$$
\dot{W}_{t, r}=-\oiint_{C S}\left(h+\frac{c^{2}}{2}\right) \rho \vec{c} \cdot \hat{n} d S+\iiint_{C V} T \rho \vec{c} \cdot \nabla s d V .
$$

Since $\dot{W}_{t, r} \geq \dot{W}_{t}$, this is the expression to be used in the calculation of the maximum power coefficient.

\section{3 | ONE-DIMENSIONAL REVERSIBLE FLUID FLOWS}

As in the derivation of the $\mathrm{BJ}$ law, $5,6,9,14$ it is assumed that the distortion of the free flow originated by the placement of the turbine can be represented by a stream tube with cylindrical symmetry surrounding it (see Figure 1). Therefore, all the air that crosses the turbine enters this tube upstream with uniform and horizontal velocity $c_{0}$, ie, the velocity of the free flow, through a planar, vertical section 0 with area $A_{0}$ and leaves it downstream through section 3-also planar and vertical with area $A_{3}$ - with uniform and horizontal velocity $c_{3}$. It is also assumed that the thermodynamic state of the air at the inlet (section 0 ) and the outlet (section 3 ) is the same and that the air enters the turbine with velocity $c_{1}$ and leaves it with velocity $c_{2}$, both uniform and horizontal. The whole stream tube and some parts of it are going to be considered as control volumes to which the laws of mechanics and thermodynamics can be applied. This will translate into relations between the thermodynamical and mechanical quantities that caractherize the flow and will allow the calculation of the maximum power coefficient.

Mass conservation implies that the mass flow $\dot{m}$ is the same through all sections of the stream tube and thus

$$
\dot{m}=\rho_{0} A_{0} c_{0}=\rho_{1} A c_{1}=\rho_{2} A c_{2}=\rho_{3} A_{3} c_{3} .
$$




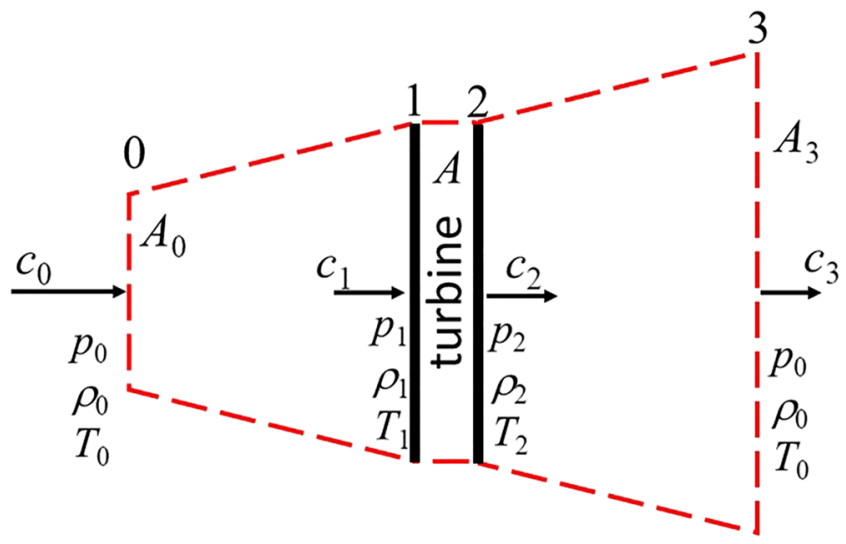

FIGURE 1 Schematic representation of the flow around the turbine. A stream tube with cylindrical symmetry around the direction of free flow is defined (region enclosed by the red dashed lines). The air flows into the stream tube by section 0 , enters the turbine in section 1 , and leaves the turbine and the stream tube at sections 2 and 3 , respectively. As a consequence, the nonvertical red dashed lines are streamlines. At each of these sections, the air is characterized by a velocity $c_{i}$ whose direction is perpendicular to the plane of the turbine (one-dimensional flow approximation) and by the thermodynamic variables $p$, pressure, $\rho$, mass density, and $T$ temperature. It is assumed that the thermodynamic states of the air when entering and exiting the stream tube are the same, ie, $\left(p_{0}, \rho_{0}, T_{0}\right)=\left(p_{3}, \rho_{3}, T_{3}\right)$ [Colour figure can be viewed at wileyonlinelibrary.com]

The turbine and the stream tube are acted on by an external force that balances the change in linear momentum and pressure of the flowing air. This balance ${ }^{14}$ (see Supporting Information) results in

$$
\dot{m}\left(c_{3}-c_{0}\right)=\left(p_{2}-p_{1}\right) A+\dot{m}\left(c_{2}-c_{1}\right)
$$

The power produced by the turbine (4) and its power coefficient will depend on the type of flow and on the equations of state of the fluid.

\section{1 | Incompressible flow}

This is the assumption made in the derivation of BJ law. The density is constant $\left(\rho_{i}=\rho\right)$ and as a consequence of mass conservation, $c_{1}=c_{2}=c$. The equation for the power produced by the turbine (4) can be simplified using the thermodynamic identity $\nabla h=T \nabla s+\nabla p / \rho^{15}$ and the continuity equation for stationary flows to obtain

$$
\dot{W}_{r, \text { inc }}=-\oiint_{C S}\left(p+\rho \frac{c^{2}}{2}\right) \vec{c} \cdot \hat{n} d S .
$$

The application to the stream tube and to the turbine gives

$$
\dot{\mathrm{W}}_{\mathrm{r}, \mathrm{inc}}=\dot{m} \frac{c_{0}^{2}-c_{3}^{2}}{2}
$$

and

$$
\dot{\mathrm{W}}_{r, \text { inc }}=\left(p_{1}-p_{2}\right) c A .
$$

The combination of these two equations imposes another relation between the flow properties and $A$

$$
\dot{m} \frac{c_{0}^{2}-c_{3}^{2}}{2}=\left(p_{1}-p_{2}\right) c A
$$

which combined with the linear momentum equation (6) (recall that $c_{1}=c_{2}=c$ ) gives

$$
c=\frac{c_{0}+c_{3}}{2}
$$

Using $\dot{m}=\rho c A=\rho A\left(c_{0}+c_{3}\right) / 2$ in (8), the power generated by the turbine is

$$
\dot{W}_{r, \text { inc }}=\rho A \frac{\left(c_{0}+c_{3}\right)^{2}\left(c_{0}-c_{3}\right)}{4}
$$

The power coefficient of the turbine in an incompressible flow is then

$$
\eta_{r, \text { inc }}(x)=\frac{(1+x)\left(1-x^{2}\right)}{2}
$$

where $x=c_{3} / c_{0}$. This function has its maximal value $\eta_{\text {inc, } \max }=16 / 27$ when $x=1 / 3$. This is BJ law.

\subsection{Isentropic flow of an ideal gas}

Instead of an incompressible fluid, as taken in the derivation of BJ law, let us consider a compressible ideal gas with constant specific heats. As usual, $C_{p}$ and $C_{V}$ are the constant pressure and constant volume specific heats, respectively, and $\gamma \equiv C_{p} / C_{V}$ is the adiabatic index. The relevant equations of state are $p=\rho r T$ (ideal gas equation), where $r=C_{p}-C_{V}$, and $\Delta h=C_{p} \Delta T$. 
For an isentropic flow $\Delta s=0$ and, for an ideal gas, $T \rho^{1-\gamma}=c$ cte. The reversible power produced by the turbine (Equation (4)) becomes

$$
\dot{W}_{r, \text { isen }}=-\oiint_{C S}\left(h+\frac{c^{2}}{2}\right) \rho \vec{c} \cdot \hat{n} d S .
$$

The application of (14) to the stream tube and to the turbine gives

$$
\dot{W}_{r, \text { isen }}=\dot{m} \frac{c_{0}^{2}-c_{3}^{2}}{2}
$$

and

$$
\dot{W}_{r, \text { isen }}=\dot{m}\left(C_{p}\left(T_{1}-T_{2}\right)+\frac{c_{1}^{2}-c_{2}^{2}}{2}\right) .
$$

Combining these equations gives

$$
\frac{c_{0}^{2}-c_{3}^{2}}{2}=C_{p}\left(T_{1}-T_{2}\right)+\frac{c_{1}^{2}-c_{2}^{2}}{2}
$$

The linear momentum equation (6) can be rewritten using the ideal gas equation and $\dot{m}=\rho_{1} c_{1} A=\rho_{2} c_{2} A$, with the result

$$
c_{0}-c_{3}=\frac{r T_{1}}{c_{1}}-\frac{r T_{2}}{c_{2}}+c_{1}-c_{2}
$$

In order to express $(17,18)$ as a function of the velocities $c_{i}$, we use the following:

1. A combination of mass conservation in the turbine, $\rho_{1} c_{1}=\rho_{2} c_{2}$, with the relation between density and temperature in the isentropic flow along the turbine, $T_{1} \rho_{1}^{1-\gamma}=T_{2} \rho_{2}^{1-\gamma}$, which holds

$$
\frac{T_{2}}{T_{1}}=\left(\frac{c_{2}}{c_{1}}\right)^{1-\gamma} .
$$

2. The definition of the Mach number $M$, for the free flow

$$
T_{0}=\frac{c_{0}^{2}}{r \gamma M_{0}^{2}} .
$$

3. Conservation of energy applied to the control volume defined by sections 0 and 1

$$
T_{1}=T_{0}+\frac{c_{0}^{2}-c_{1}^{2}}{2 C_{p}}=\frac{c_{0}^{2}}{r \gamma M_{0}^{2}}+\frac{c_{0}^{2}-c_{1}^{2}}{2 C_{p}}
$$

Using (19) and (21) to eliminate $T_{1}$ and $T_{2}$ from (17) and (18), these equations become a relation between the velocities $c_{i}$ and the Mach number of the free flow, $M_{0}$

$$
\begin{aligned}
& 1-x^{2}=\left(\frac{2}{(\gamma-1) M_{0}^{2}}+1-y^{2}\right)\left(1-z^{1-\gamma}\right)+y^{2}\left(1-z^{2}\right), \\
& (1-x) y=\left(\frac{1}{\gamma M_{0}^{2}}+\frac{\gamma-1}{2 \gamma}\left(1-y^{2}\right)\right)\left(1-z^{-\gamma}\right)+y^{2}(1-z),
\end{aligned}
$$

where $x=c_{3} / c_{0}, y=c_{1} / c_{0}$, and $z=c_{2} / c_{1}$. These equations allow the calculation of $y$ and $z$ as a function of $x$ for a given $M_{0}$. The power coefficient of the turbine may be written, using (15) and $\dot{m}=\rho_{1} c_{1} A$, as

$$
\eta_{r, \text { isen }}=\frac{\rho_{1} c_{1}}{\rho_{0} c_{0}}\left(1-\frac{c_{3}^{2}}{c_{0}^{2}}\right) .
$$

Using the relation for the isentropic flow $\rho_{1} / \rho_{0}=\left(T_{1} / T_{0}\right)^{\frac{1}{\gamma-1}},(21)$ and the definitions of $x$ and $y$, the power coefficient of the turbine in an isentropic flow of an ideal gas is obtained as a function of $x$ and $M_{0}$ (for a given $\gamma$ )

$$
\eta_{r, \text { isen }}\left(x, M_{0}\right)=\left(1+\frac{\gamma-1}{2} M_{0}^{2}\left(1-y^{2}\right)\right)^{\frac{1}{\gamma-1}} y\left(1-x^{2}\right)
$$

Notice that $y$ is a function of $x$ and $M_{0}$ given by Equations (22) and (23) that may be computed numerically for the general case.

However, a perturbative analysis gives us also interesting results. To first order in $M_{0}^{2}$, we have

$$
y\left(x, M_{0}^{2}\right)=\frac{1+x}{2}-\frac{M_{0}^{2}}{8}(1-x)(1+x)^{2}+\ldots
$$

The zeroth order term leads to the Betz power coefficient (see Equation (13)), whose maximum value $\eta_{\text {inc, } \max }=16 / 27$ is attained when $x=1 / 3$. To first order in $M_{0}^{2}$, the maximum power coefficient (also for $x=1 / 3$ ) is given by 


$$
\eta_{r, \text { isen, } \max }=\frac{16}{27}+\frac{8}{243} M_{0}^{2}+\ldots
$$

We notice that this result does not depend on $\gamma$, but only on $M_{0}$.

\subsection{Isothermal flow of an ideal gas}

For an isothermal flow $(T=\mathrm{cte})$ of an ideal gas, the reversible power produced by the turbine (Equation (4)) is

$$
\dot{W}_{r, \text { isot }}=-\oiint_{C S}\left(\frac{c^{2}}{2}-T s\right) \rho \vec{c} \cdot \hat{n} d S .
$$

Applying this equation to the stream tube and the turbine gives

$$
\begin{gathered}
\dot{\mathrm{W}}_{r, \text { isot }}=\dot{m} \frac{c_{0}^{2}-c_{3}^{2}}{2} . \\
\dot{\mathrm{W}}_{r, \text { isot }}=\dot{m} T\left(s_{2}-s_{1}\right)+\dot{m} \frac{c_{1}^{2}-c_{2}^{2}}{2} .
\end{gathered}
$$

In an ideal gas with constant specific heats, the specific entropy variation in an isothermal process is $s_{2}-s_{1}=r \ln \frac{p_{1}}{p_{2}}=r \ln \frac{\rho_{1}}{\rho_{2}}$. Conservation of mass in the turbine reads $\rho_{1} c_{1}=\rho_{2} c_{2}$, leading to

$$
\dot{\mathrm{W}}_{\text {isot }}=\dot{m} r T \ln \frac{c_{2}}{c_{1}}+\dot{m} \frac{c_{1}^{2}-c_{2}^{2}}{2},
$$

which, combined with (29), gives

$$
\frac{c_{0}^{2}-c_{3}^{2}}{2}=r T \ln \frac{c_{2}}{c_{1}}+\frac{c_{1}^{2}-c_{2}^{2}}{2} .
$$

After considering that $\dot{m}=\rho_{1} c_{1} A=\rho_{2} c_{2} A$ and $p_{i} / \rho_{i}=r T$, the linear momentum equation (6) for an isothermal flow can be expressed as

$$
c_{0}-c_{3}=r T\left(\frac{1}{c_{1}}-\frac{1}{c_{2}}\right)+c_{1}-c_{2}
$$

Equations (32) and (33) can be expressed as

$$
\begin{gathered}
\left(1-x^{2}\right) \gamma M_{0}^{2}=2 \ln z+\gamma M_{0}^{2} y^{2}\left(1-z^{2}\right), \\
\gamma M_{0}^{2} y z(1-x-y(1-z))=z-1,
\end{gathered}
$$

where, as before, $x=c_{3} / c_{0}, y=c_{1} / c_{0}, z=c_{2} / c_{1}$, and $r T=c_{0}^{2} / \gamma / M_{0}^{2}$. These two equations allow the calculation of $y, z$ for fixed $x, M_{0}$.

The power coefficient for an isothermal flow is then

$$
\eta_{r, \text { isot }}=\frac{\rho_{1} A c_{1}\left(c_{0}^{2}-c_{3}^{2}\right)}{\rho_{0} A c_{0}^{3}}=\frac{\rho_{1}}{\rho_{0}} y\left(1-x^{2}\right) .
$$

In order to determine $\rho_{1} / \rho_{0}$ as a function of the velocities $c_{i}$, we use the conservation of energy for the flow between sections 0 and 1 of the stream tube to obtain

$$
\frac{c_{1}^{2}-c_{0}^{2}}{2}=T\left(s_{1}-s_{0}\right)=-r T \ln \frac{\rho_{1}}{\rho_{0}}
$$

from which

$$
\frac{\rho_{1}}{\rho_{0}}=\exp \left(\frac{c_{0}^{2}-c_{1}^{2}}{2 r T}\right) .
$$

The power coefficient can then be expressed as a function of $x$ and $M_{0}$,

$$
\eta_{r, \text { isot }}\left(x, M_{0}\right)=\exp \left(\frac{\gamma M_{0}^{2}}{2}\left(1-y^{2}\right)\right) y\left(1-x^{2}\right)
$$

with $y\left(x, M_{0}\right)$ given by equations (34) and (35). The perturbative analysis leads to the same maximum power coefficient as is the isentropic case, to first order in $M_{0}^{2}$ (it had been shown already that it did not depend on $\gamma$ )-Equation (27).

\subsection{Power coefficient calculations}

The power produced by the turbine (4) and its power coefficient will depend on the type of flow and on the equations of state of the fluid. We have combined (4), (5), and (6) in three cases: (i) incompressible flow ( $\rho=$ cte), (ii) isentropic flow ( $s=$ cte) and (iii) isothermal flow ( $T=$ cte) of an ideal gas. In case (i), the BJ law is recovered: The power coefficient is a function of the ratio $x=c_{3} / c_{0}$ only and is given by a single expression, $\eta_{r, \text { inc }}=(1+x)\left(1-x^{2}\right) / 2$ (Equation (13)). In cases (ii) and (iii), we obtain $\eta_{r, \text { isen }}\left(x, M_{0}, \gamma\right)$ and $\eta_{r, \text { isot }}\left(x, M_{0}, \gamma\right)$ : The efficiencies depend not only on $x$ 

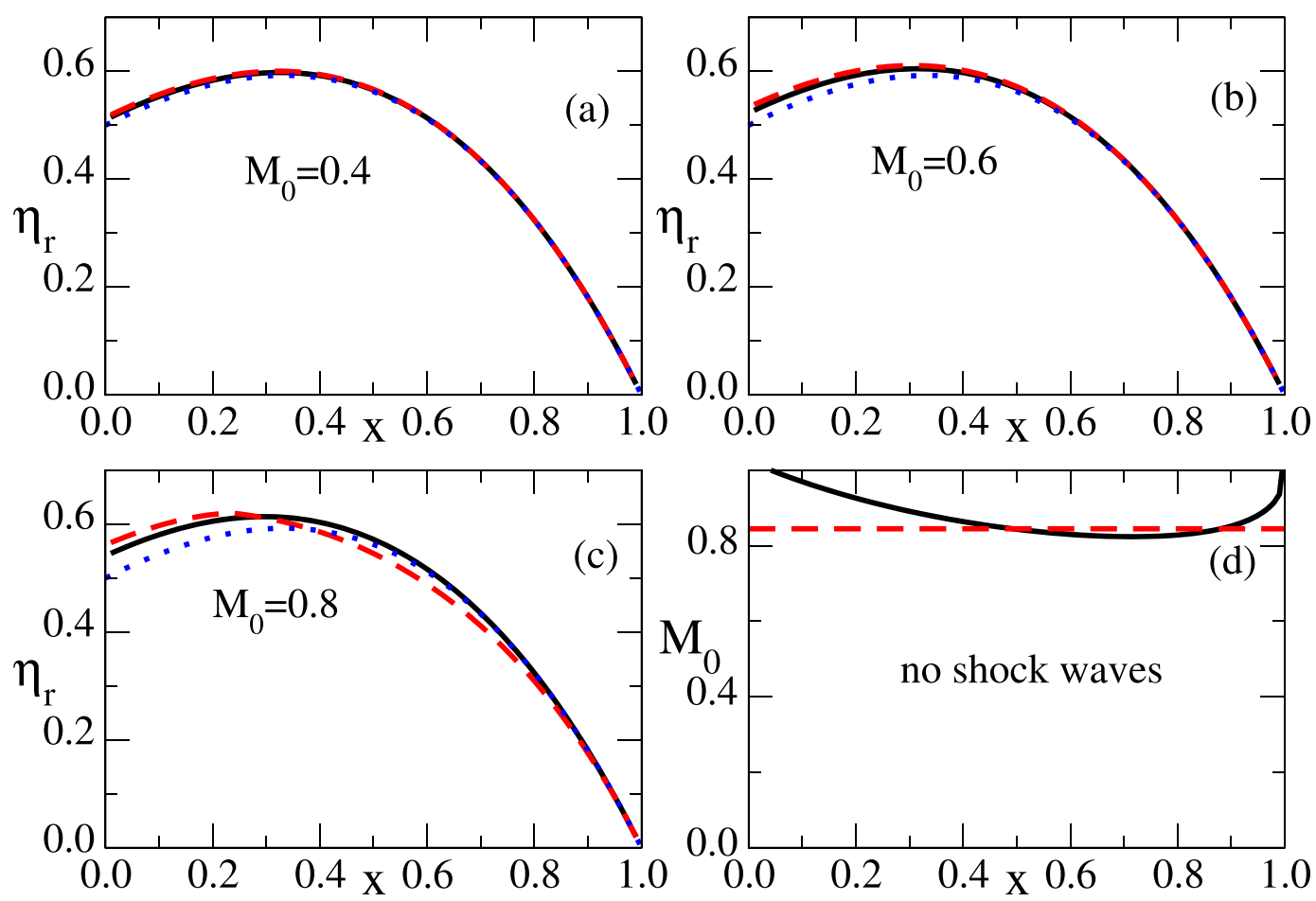

FIGURE 2 (A) to (C): reversible power coefficient $\eta_{r}$ as a function of $x=c_{3} / c_{0}$ for incompressible (blue dotted line), isentropic (black full line), and isothermal (red dashed line) flow, for the indicated values of $M_{0}$, the Mach number of the free flow; (D): for the pairs of values ( $x$, $\left.M_{0}\right)$ above the black full (red dashed) line, isentropic (isothermal reversible) flow is no longer possible due to the formation of shock waves. The isentropic and isothermal flows are that of an ideal gas with adiabatic index $\gamma=1.4$ [Colour figure can be viewed at wileyonlinelibrary.com]

but also on the Mach number of the free flow, $M_{0}$, and on the adiabatic index of the gas $\gamma$. To calculate them, we must solve a system of three equations ((22), (23), and (25)) in case (ii) and ((34), (35), and (39)) in case (iii)).

The efficiencies for these three flows are computed as a function of $x$ and shown in Figure 2A to $C$ for three values of $M_{0}$ (and for $\gamma=1.4$ ). For low values of $M_{0}$ (up to $\approx 0.5$ ), the efficiencies are almost indistinguishable: This means that the density variations are negligible, and the incompressible approximation holds. For higher values of $M_{0}$, the efficiencies become noticeable different: The flow is in the subsonic regime, where density variations are important, but shock waves are not present. For values of $M_{0}$ close to the supersonic regime (larger than $\approx 0.8$ ), it is not possible to compute $\eta_{r}$ for all values of $x$, since the assumption of reversibility becomes invalid when shock waves appear in some part of the flow. For each type of compressible flow, the plane $\left(x, M_{0}\right)$ is divided (for a given $\gamma$ ) into two regions (see Figure 2D): Only in the no shock wave region is it possible to define $\eta_{r}$. In this region of parameters, all $M_{i}$ (Mach number of the flow in section 1 of the CV-see Figure 1 ) are $<1$ for isentropic flows and $<1 / \sqrt{\gamma}$ for isothermal flows. The lines in Figure 2D were calculated: (a) for isentropic flows by solving $M_{2}=1$, since $M_{2}$ is always the largest Mach number; (b) for isothermal flows by setting $M_{0}=1 / \sqrt{\gamma}$, since $M_{i}<1 / \sqrt{\gamma}$ when $M_{0}<1 / \sqrt{\gamma}$.

The maximum power coefficient of turbines in each of the three cases is obtained by determining the maximum of the functions $\eta_{r}(x)$. In the incompressible case, this calculation is done analytically, and the BJ limit is recovered: $\eta_{\text {inc, } \max }=16 / 27$. The results of the numerical calculation for the maximum power coefficient of isentropic $\left(\eta_{\text {isen,max }}\left(M_{0}\right)\right)$ and isothermal $\left(\eta_{\text {isot,max }}\left(M_{0}\right)\right)$ turbines for each value of $M_{0}$ are plotted in Figure 3

FIGURE 3 Maximum power coefficient of isentropic (full black line) and isothermal (dashed red line) turbines compared with the Betz limit (incompressible case, $\eta_{\text {inc, } \max }=16 / 27$ ) as a function of the Mach number of free flow, $M_{0}$. The green dotted line represents the Taylor expansion of $\eta_{\max } / \eta_{\text {inc,max }}$ to first order in $M_{0}^{2}$ (see the text) [Colour figure can be viewed at wileyonlinelibrary.com]

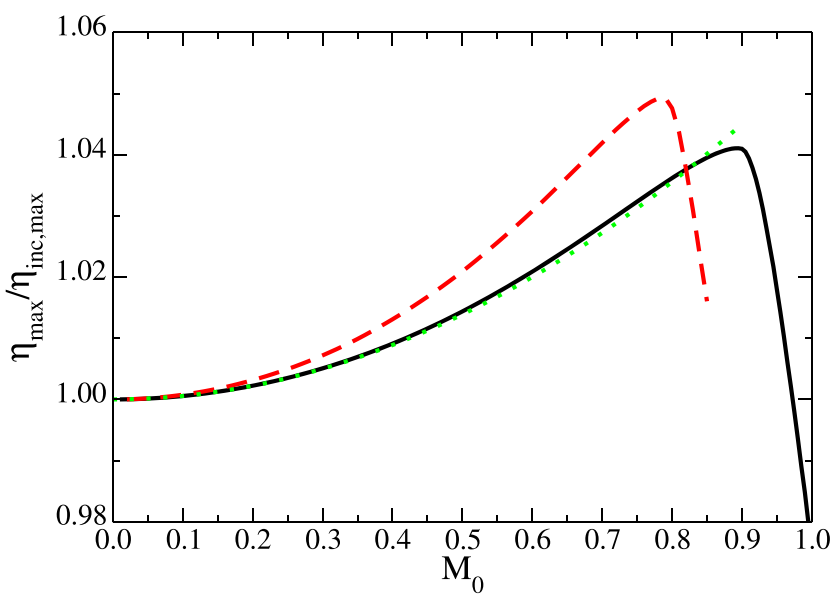



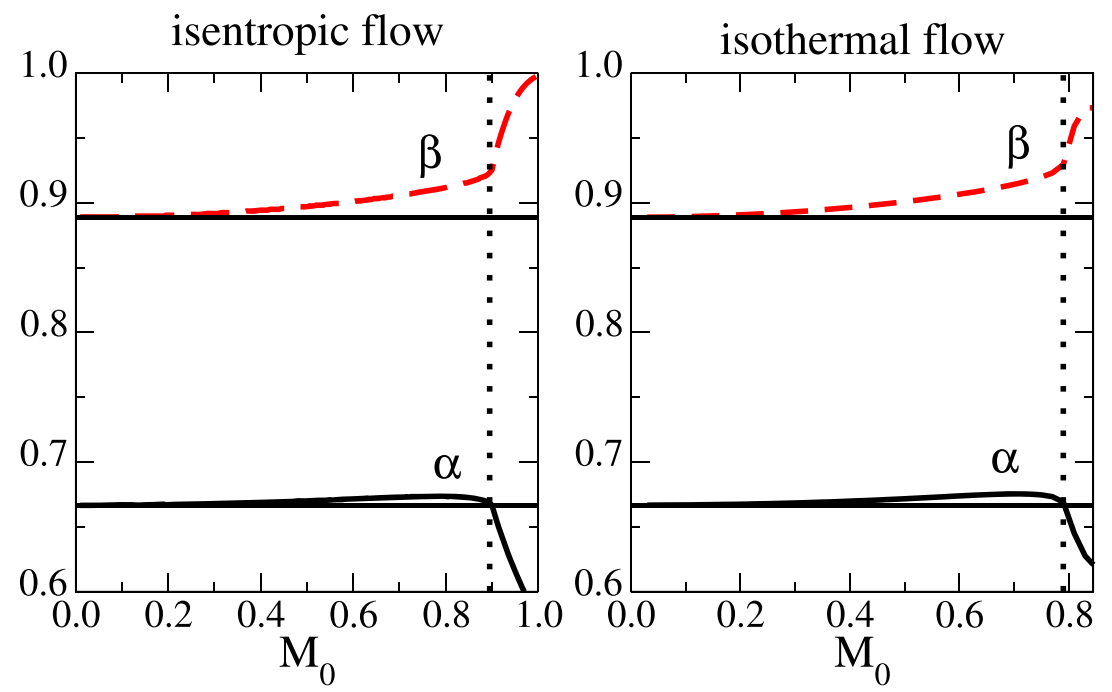

FIGURE 4 Components $\alpha$ (40) and $\beta$ (41) of the maximum power coefficient of wind turbines. The horizontal full lines are the values $\alpha=2 / 3$ and $\beta=8 / 9$ for the incompressible case. The dotted vertical lines mark the value of $M_{0}$ for which the power coefficient (the product $\alpha \times \beta$ ) is maximum [Colour figure can be viewed at wileyonlinelibrary.com]

and compared with $\eta_{\text {inc,max }}$. The maximum efficiencies in these cases are larger than 16/27 at all values of $M_{0}$ in the isothermal case and for $M_{0}$ up to $\approx 0.95$ in the isentropic case. At high Mach numbers of the free flow $\left(M_{0} \approx 0.9\right.$ for isentropic flows and $M_{0} \approx 0.8$ for isothermal flows), the power coefficient is increased by $4-5 \%$. The perturbative expansion to first order in $M_{0}^{2}, \eta_{r \text {,isen,max }} \approx \eta_{r, \text { isot,max }} \approx 16 / 27+8 / 243 M_{0}^{2}$ is also shown. Notice that this quadratic expression, valid for small values of $M_{0}$, follows approximately the maximum efficiency for the isentropic case up to $M_{0}<0.8$. These results suggest a reinterpretation of the $B J$ limit as a convenient, practical, and easily derivable value for maximum wind turbine power coefficient, that is, always surpassed when compressibility is taken into account.

\section{5 | Analysis}

The origin of the increase in power coefficient due to compressibility can be better understood when the power coefficient is considered as a product of two factors, $\eta_{r}=\alpha \times \beta$. $\alpha$ is the ratio between the mass flow that crosses the turbine and the mass flow of the wind

$$
\alpha=\frac{\rho_{1} c_{1}}{\rho_{0} c_{0}}
$$

$\beta$ is the fraction of energy per unit mass of fluid that the turbine extracts from the free flow,

$$
\beta=1-\frac{c_{3}^{2}}{c_{0}^{2}}=1-x^{2}
$$

The maximum power coefficient in incompressible flow is obtained when $\alpha_{\text {inc }}=2 / 3$ and $\beta_{\text {inc }}=8 / 9$ : The mass flow arriving to the turbine is $2 / 3$ of that of free flow; the turbine then converts into work $8 / 9$ of the kinetic energy contained in each unit mass of the fluid. Figure 4 shows the results for $\alpha$ and $\beta$ in compressible flows, in conditions of maximum power coefficient as a function of $M_{0}$. The behavior is similar in both isentropic and isothermal flows: $\beta$ grows continuously with increasing $M_{0}$ while $\alpha$ has a slower increase and a sudden decrease at high $M_{0}$. Except in the case of $\alpha$ at very large $M_{0}$, both coefficients are larger than those of incompressible flow. Therefore, the power coefficient of compressible flows is larger than that of the incompressible limit, because compressibility leads to a larger amount of fluid arriving to the turbine ( $\alpha>2 / 3$ ) and, specially, to the ability of the turbine to extract more work from the flow $(\beta>8 / 9)$. Notice that an increase over the Betz limit in power coefficient can also be obtained in incompressible flow by surrounding the turbine with a diffuser ${ }^{16}$ (the so called diffuser augmented wind turbines [DAWT]). However, in DAWT's, this increase is solely due to an increase in $\alpha .{ }^{16}$ On the contrary, the largest contribution to the increase of the maximum power coefficient in compressible flow comes from the increase in $\beta$, ie, from the increase in the efficiency of the turbine when converting the actual wind kinetic energy that reaches it into work (see Figure 4).

\section{4 | CONCLUSION}

The analysis presented in this work clarifies the status of the BJ limit: It is a theoretical upper bound for the power coefficient of reversible wind turbines within the incompressibility hypothesis. Once this hypothesis is dropped, larger efficiencies can be attained. The upper limit will depend on the type of flow and on the equation of state of the fluid. Our results also strongly suggest that the BJ limit is in fact the smallest maximum possible power coefficient, since it appears to be reached from above in the limit $M_{0} \rightarrow 0$ of maximum efficiencies of compressible flows. Even if these results are expected to have small practical value for the real systems in current use, they clarify the role of general scientific principles (like the first and second law of thermodynamics) in the energy conversion occurring in wind turbines. The new results of this paper are condensed in 
the simple form of a series expansion of the maximum power coefficient to first order in $M_{0}^{2}, \eta_{\max }=16 / 27+8 / 243 M_{0}^{2}$, valid for $M_{0}$ up to $\approx 0.8$ and for ideal isentropic gas flows. This simple equation contains the $B J$ limit (recovered when $M_{0}=0$ ) and shows that compressibility increases the efficiency but only a few percent when compared with the incompressible case.

Notice that the role of compressibility has been neglected in the study of real (irreversible) wind turbines, since they operate at low air velocities (eg, they are shut down when wind velocity exceeds $\approx 20 \mathrm{~m} / \mathrm{s}^{6}$ or $M_{0} \approx 0.06$ ). However, at the tip of the large and more modern wind turbines blades, air can reach velocities that correspond to $M_{0} \approx 0.2-0.3$ and so the effects of compressibility on the wake of the turbine and on its power production are starting to be explored through more realistic models and more sophisticated numerical methods. ${ }^{17}$ Although one should not forget that for higher Mach numbers, turbulence effects increase the irreversibility of the flow, it would be important to extend the present analysis to a model that considers the rotation of the turbine and its size, ie, the actuator disk is replaced by a rotor that creates a wake in the air flow. ${ }^{6,14,18}$ The power coefficient of reversible turbines with a given tip speed ratio-the ratio of the velocity of the tip of the turbine to the wind velocity-in compressible flows should be a reference for more realistic irreversible flows. It could also be compared with the known results for the incompressible case.

\section{ACKNOWLEDGEMENTS}

We thank P.I.C. Teixeira and N.A.M. Araújo for a critical reading of this manuscript. This work was partially funded by the Portuguese Science Foundation (FCT) through project UID/FIS/00618/2019.

\section{ORCID}

J. M. Tavares (iD) https://orcid.org/0000-0002-1509-9707

P. Patrício (D) https://orcid.org/0000-0002-9050-9956

\section{REFERENCES}

1. Callen HB. Thermodynamics and an Introduction to Thermostatistics, 2nd ed. New York: John Wiley and Sons; 1985.

2. Çengel YA, Boles MA. Thermodynamics: An Engineering Approach, 8th ed. New York: McGraw Hill; 2015.

3. Global Wind Report. Global Wind Energy Council. https://gwec.net/; 2017.

4. Okulov VL, van Kuik GA. The Betz-Joukowsky limit: on the contribution to rotor aerodynamics by the British, German and Russian scientific schools. Wind Energ. 2012;15:335-344.

5. Betz A. The maximum of the theoretically possible exploitation of wind by means of a wind motor. Wind Eng. 2013;37:441-446. Translation of Zeitschrift fr das gesamte Turbinenwesen 26, 307 (1920), by H. Hamann, J. Thayer and A.P. Schaffarczyk.

6. Manwell JF, McGowan JG, Rogers AL. Wind Energy Explained: Theory, Design and Application. West Sussex: John Wiley \& Sons; 2009.

7. Hansen MOL. Aerodynamics of Wind Turbines, 2nd ed. West Sussex: Earthscan; 2008.

8. Burton T, Sharpe D, Jenkins N, Bossanyi E. Wind Energy. Handbook. London: John Wiley and Sons; 2001.

9. White FM. Fluid Mechanics. 7th ed. New York: McGrawHill; 2011.

10. Huleihila M. A comparative analysis of deep level emission in ZnO layers deposited by various methods. J of Appl Phys. 2009;105:104908.

11. Vogeley AW. Axial-momentum theory for propellers in compressible flow. NACA Technical Note 2164, Langley Field, VA, United States, National Advisory Committee for Aeronautics. Langley Aeronautical Lab.; 1951.

12. Delano JB. Crigler, JL. NACA RM L53A07; 1953.

13. Oo HHN. Actuator disk theory for compressible flow. MSC thesis: California Polytechnic State University (USA); 2017.

14. Glauert H. Airplane Propellers. In: Durand WF, ed. Aerodynamic Theory. Berlin: Springer Verlag; 1935:169-360.

15. Landau LD, Lifshitz EM. Fluid Mechanics. 2nd ed. Oxford: Pergamon Press; 1987.

16. Hjort S, Larsen H. A multi-element diffuser augmented wind turbine. Energies. 2014;7:3256-3281.

17. Yan C, Archer CL. Assessing compressibility effects on the performance of large horizontal-axis wind turbines. Applied Energy. 2018;212:33-45.

18. Hunsaker DF, Philips WF. Momentum theory with slipstream rotation applied to wind turbines. In: 31st AIAA Applied Aerodynamics Conference; 2013; San Diego, California: AIAA-2013-3161.

\section{SUPPORTING INFORMATION}

Additional supporting information may be found online in the Supporting Information section at the end of the article.

How to cite this article: Tavares JM, Patricio P. Maximum thermodynamic power coefficient of a wind turbine. Wind Energy. 2020;23:

1077-1084. https://doi.org/10.1002/we.2474 